\title{
Fibrosis endomiocárdica: una revisión actualizada a propósito de unos casos en un programa de falla cardiaca
}

\author{
Endomyocardial fibrosis: an updated review, about some cases in a heart failure program
}

\author{
Cristhian F. Ramírez-Ramos ${ }^{1 *}$, Catalina Gallegoº ${ }^{2}$ Luis Fajardo², Adriana Agudelo ${ }^{3}$ y \\ Clara I. Saldarriaga-Giraldo ${ }^{3,5}$
}

${ }^{1}$ Departamento de Cardiología Intervencionista y Hemodinámica, Universidad Pontificia Bolivariana y Clínica cardioVID; ${ }^{2}$ Departamento de Cardiología Clínica y Clínica de Falla Cardiaca, Universidad Pontificia Bolivariana y Clínica cardioVID; ${ }^{3}$ Departamento de Enfermería y Clínica de Falla Cardiaca, Clínica cardioVID; ${ }^{4}$ Departamento de Cardiología, Universidad de Antioquia. Medellín, Colombia

\section{Resumen}

Introducción: La fibrosis endomiocárdica es una enfermedad cardiovascular olvidada, principal causa de cardiomiopatía restrictiva. Se estima que en el mundo hay 10-12 millones de personas con la enfermedad. La mayoría se restringen a zonas de África, Asia y, en Latinoamérica, en Brasil y Colombia. La etiología y la fisiopatología son poco comprendidas. Para su diagnóstico se deben tener en cuenta los datos del paciente, el cuadro clínico y los hallazgos ecocardiográficos. Presentamos los casos confirmados en un centro de referencia cardiovascular. Descripción de los casos: Se han documentado tres casos, de los cuales dos son mujeres. Todos debutaron con un cuadro insidioso de disnea con esfuerzo. Los hallazgos ecocardiográficos fueron disfunción diastólica con patrón restrictivo, insuficiencia moderada de válvulas auriculoventriculares e hipertensión pulmonar; en dos casos se encontraron imágenes anormales endocárdicas en el ventrículo izquierdo y se realizó biopsia endomiocárdica para confirmar el diagnóstico. Ninguno mejoró la clase funcional ni la frecuencia de hospitalizaciones. Conclusiones: La fibrosis endomiocárdica debe considerarse en todo paciente con falla cardiaca con un patrón restrictivo asociado y con poca respuesta al manejo farmacológico convencional. Los pacientes presentados tenían hallazgos típicos de la enfermedad, con la particularidad de ser de edades mayores.

Palabras clave: Falla cardiaca. Fibrosis endomiocárdica. Eosinofilia. Cardiomiopatía restrictiva.

\section{Abstract}

Introduction: Endomyocardial fibrosis is one of the neglected cardiovascular disease, the main cause of restrictive cardiomyopathy. There are an estimated 10-12 million people with the disease worldwide. Most are restricted to areas of Africa, Asia and in Latin America in Brazil and Colombia. The etiology and pathophysiology are poorly understood. For its diagnosis, the patient's data, the clinical picture and the echocardiographic findings must be taken into account. We present the confirmed cases in a cardiovascular referral center. Description of the cases: Three cases have been documented of which two are women. All debuted with insidious dyspnea on exertion. The echocardiographic findings were diastolic dysfunction with a restrictive pattern, moderate atrioventricular valve insufficiency, and pulmonary hypertension. In two cases, endocardial abnormal images were found in the left ventricle and an endomyocardial biopsy was performed to confirm the diagnosis. None improved functional class or frequency of hospitalizations. Conclusions: Endomyocardial fibrosis should be considered in a

\section{Correspondencia:}

*Cristhian F. Ramírez-Ramos

E-mail: cristhianramos1989@ hotmail.com
Fecha de recepción: 02-07-2020

Fecha de aceptación:17-12-2020

DOI: 10.24875/RCCAR.M21000094
Disponible en internet: 07-01-2022 Rev Colomb Cardiol. 2021;28(6):530-538 www.rccardiologia.com 0120-5633 / C 2020 Sociedad Colombiana de Cardiología y Cirugía Cardiovascular. Publicado por Permanyer. Este es un artículo open access bajo la licencia CC BY-NC-ND (http://creativecommons.org/licenses/by-nc-nd/4.0/). 
patient with heart failure with an associated restrictive pattern, and with little response to conventional pharmacological management. The patients presented had typical findings of the disease with the particularity of being older.

Key words: Heart failure. Endomyocardial fibrosis. Eosinophilia, Restrictive cardiomyopathy.

\section{Introducción}

La fibrosis endomiocárdica es una enfermedad cardiovascular emergente, relacionada con importante discapacidad y muertes prematuras en los países endémicos. Es la cardiomiopatía restrictiva más común en el mundo, con grupos geográficos definidos y una importante relación con la pobreza, lo que ha generado bloqueos en el progreso necesario de esta condición; en la actualidad existe aún un vacío en nuestro conocimiento en cuanto a su etiología, patogénesis y evolución natural.

Es también una causa importante de cardiomiopatía en jóvenes, casi la mitad de los casos se presentan en África Subsahariana y de manera menos común en el sur de Asia y América Latina, regiones por lo general con acceso limitado a los servicios de atención, por lo que se cree que hay un gran subdiagnóstico. El reconocimiento de estos pacientes es la mayoría de las veces en hospitales de mayor complejidad y cuando hay signos de enfermedad avanzada con complicaciones estructurales o hemodinámicas graves, etapas en que el manejo es poco efectivo.

Presentamos los casos de pacientes de un programa de insuficiencia cardiaca en un centro cardiovascular de referencia, resaltando las particularidades del comportamiento en nuestra región y la utilidad de algunas ayudas diagnósticas, como la biopsia endomiocárdica, en el algoritmo de trabajo de la falla cardiaca.

\section{Descripción de los casos}

\section{Caso 1}

Mujer de 72 años, natural y residente de Medellín, ama de casa, quien ingresa al hospital por un cuadro clínico de 8 días de evolución de disnea en progresión de moderados a pequeños esfuerzos, con ortopnea, astenia, adinamia, sin edema de miembros inferiores ni disnea paroxística nocturna. Como antecedentes de importancia, la paciente tenía historia de hipertensión arterial crónica, trastorno afectivo bipolar e insomnio. Previamente había tenido una hospitalización por causa no cardiovascular (cuadro convulsivo) en otro centro médico de la ciudad, donde le realizaron estudios cardiovasculares con evidencia de una insuficiencia mitral moderada-grave, insuficiencia aórtica moderada y descripción de una imagen densa en el ventrículo izquierdo interpretada como un trombo intracavitario. También con probabilidad alta hipertensión pulmonar. En la exploración física de ingreso a urgencias se encontró con congestión pulmonar importante, desaturación e hipoxemia, requiriendo traslado a la unidad de cuidado coronario considerando una falla cardiaca descompensada con un perfil hemodinámico caliente y húmedo. Durante la estancia hospitalaria, la paciente presenta progresión de la sintomatología con empeoramiento de la congestión pulmonar y necesidad de soporte ventilatorio no invasivo. Los estudios institucionales mostraron un hemograma con eosinofilia y en el ecocardiograma una masa ecogénica (mayor ecogenicidad que los tejidos blandos) sobre el borde endocárdico, contráctil, con características de corresponder a una fibrosis endomiocárdica (Fig. 1); las valvulopatías se clasificaron como moderadas, y además con una función diastólica con características de patrón restrictivo y una probabilidad alta de hipertensión pulmonar (Tabla 1). Se consideró que la etiología de la falla cardiaca no era valvular. En el score de Mucumbi se encontraron tres criterios mayores (8 puntos) y dos criterios menores (4 puntos), con una clasificación de gravedad de la enfermedad de moderada. La paciente presentó además compromiso de la función renal, lo que limitó la posibilidad de realizar una resonancia magnética cardiaca en búsqueda de la etiología (se había descartado también enfermedad coronaria) (Tabla 1), así que se solicitó realizar un cateterismo cardiaco derecho con biopsia endomiocárdica, procedimiento efectuado sin complicaciones cuando las condiciones de la paciente eran estables. El reporte de la biopsia mostró zonas de aumento endocárdico con infiltrado inflamatorio y sin compromiso de miocardio, cambios sugestivos de una fibrosis endomiocárdica (Tabla 1).

\section{Caso 2}

Mujer de 68 años, procedente de Bello Antioquia, de ocupación hogar, en seguimiento en la clínica de falla cardiaca con una fracción de eyección en rango intermedio diagnosticada, con un cuadro de disnea progresiva y signos congestivos periféricos y pulmonares, como evidencia de una fibrilación auricular crónica sospechando etiología de posible origen arrítmico/ 


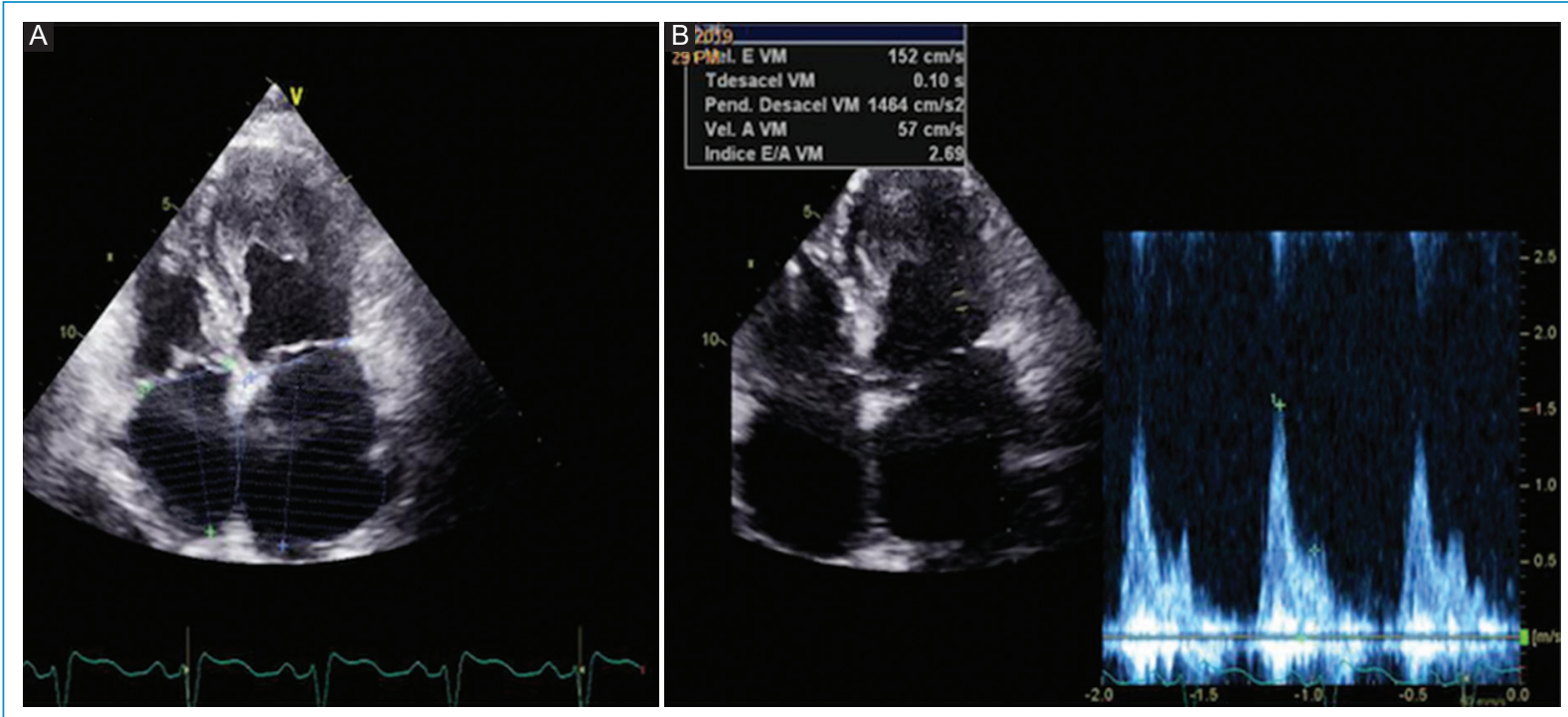

Figura 1. Ecocardiograma del caso 1. A: vista apical de cuatro cámaras donde se aprecia la ocupación biapical con predominio izquierdo a nivel de los segmentos medio y apical. B: influjo de la válvula mitral donde de aprecia un patrón restrictivo, con relación E/A 2.69 y con un tiempo de desaceleración de la onda E de 100 ms.

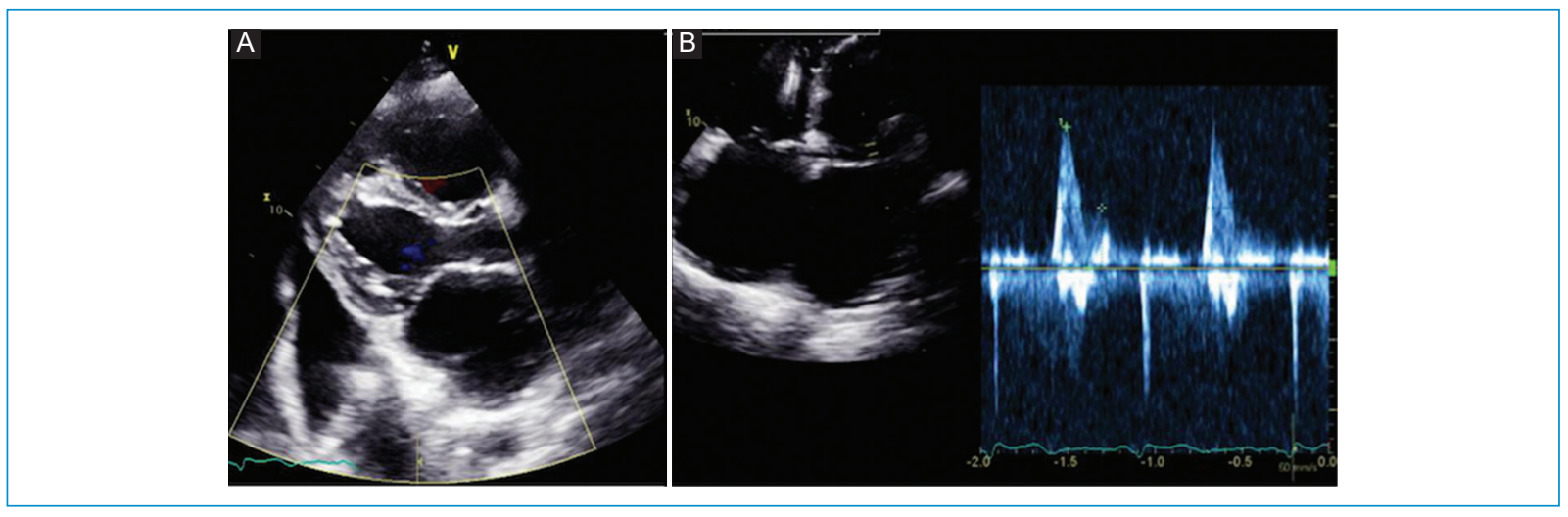

Figura 2. Ecocardiograma del caso 2. A: vista en el eje largo paraesternal donde se aprecia un realce endocárdico del ventrículo izquierdo. B: influjo mitral con un patrón restrictivo, con una relación de onda E/A de 2.98 y un tiempo de desaceleración de E de $130 \mathrm{~ms}$.

taquicardiomiopatía (Tabla 1). Como antecedentes tenía una neumopatía crónica mixta obstructiva/restrictiva en contexto de extabaquismo y síndrome de apnea obstructiva del sueño grave, así como también hipertensión arterial sistémica crónica. Durante la evolución y el seguimiento ecocardiográfico mostró dilatación importante de cavidades auriculares, disfunción diastólica de tipo restrictivo, hipertensión pulmonar y sin cambios sugestivos de una fibrosis endomiocárdica. En el score de Mucumbi se encontraron un criterio mayor (8 puntos) y dos criterios menores (4 puntos), con una clasificación de gravedad de la enfermedad de leve (Fig. 2).
Se planteó la necesidad de realizar una resonancia magnética cardiaca, pero por imposibilidad de efectuar apneas por parte de la paciente a la fecha no se ha podido practicar dicho estudio. Sin embargo, ante la evolución clínica y las características ecocardiográficas se considera que la etiología de su falla cardiaca es una fibrosis endomiocárdica.

\section{Caso 3}

Varón de 47 años, natural y procedente de Antioquia, de ocupación oficios varios, quien presentó un cuadro 
Tabla 1. Características clínicas, paraclínicas y de seguimiento de los pacientes con fibrosis endomiocárdica

\begin{tabular}{|c|c|c|c|}
\hline Parámetro & Paciente 1 & Paciente 2 & Paciente 3 \\
\hline Edad & 72 años & 47 años & 68 años \\
\hline Sexo & Mujer & Hombre & Mujer \\
\hline IMC & 28.7 & 23.6 & 27.2 \\
\hline Síntomas al diagnóstico & Disnea de esfuerzo & $\begin{array}{l}\text { Disnea de esfuerzo, } \\
\text { palpitaciones y fibrilación } \\
\text { auricular }\end{array}$ & $\begin{array}{l}\text { Disnea de esfuerzo y edemas } \\
\text { periféricos }\end{array}$ \\
\hline $\begin{array}{l}\text { Clase funcional NYHA al } \\
\text { diagnóstico }\end{array}$ & II & III & III \\
\hline $\begin{array}{l}\text { Clase funcional NYHA al } \\
\text { seguimiento }\end{array}$ & II & II & III \\
\hline $\begin{array}{l}\text { Cuadro hemático y conteo } \\
\text { diferencial inicial }\end{array}$ & $\begin{array}{l}\text { Leucocitos } 7440 / \mathrm{mm}^{3} \\
\text { Neutrófilos } 51 \% \text {, conteo } 3839 / \mathrm{mm}^{3} \\
\text { Linfocitos } 23 \% \text {, conteo } 1763 / \mathrm{mm}^{3} \\
\text { Eosinófilos } 15.3 \% \text {, conteo } 1138 / \\
\mathrm{mm}^{3} \\
\text { Hemoglobina } 8.9 \mathrm{~g} / \mathrm{dl} \\
\text { VCM } 98 \mathrm{fl} \\
\text { HCM } 30.5 \mathrm{pg} \\
\text { Plaquetas } 104,000 / \mathrm{mm}^{3}\end{array}$ & $\begin{array}{l}\text { Leucocitos } 8450 / \mathrm{mm}^{3} \\
\text { Neutrófilos } 79 \% \\
\text { Linfocitos } 16 \% \\
\text { Eosinófilos } 462 / \mathrm{mm}^{3} \\
\text { Hematocrito } 34.9 \% \\
\text { Hemoglobina } 11.3 \mathrm{~g} / \mathrm{dl} \\
\text { RDW } 15.5 \\
\text { Plaquetas } 257,000 / \mathrm{mm}^{3}\end{array}$ & $\begin{array}{l}\text { Leucocitos } 6950 / \mathrm{mm}^{3} \\
\text { Neutrófilos } 57 \% \text {, conteo } \\
3756 / \mathrm{mm}^{3} \\
\text { Linfocitos } 35 \% \text {, conteo } \\
2307 / \mathrm{mm}^{3} \\
\text { Eosinófilos } 1 \% \text {, conteo } 66 / \mathrm{mm}^{3} \\
\text { Hemoglobina } 12.6 \mathrm{~g} / \mathrm{dl} \\
\text { VCM } 88 \mathrm{fl} \\
\text { HCM } 27.8 \mathrm{pg} \\
\text { Plaquetas } 203,000 / \mathrm{mm}^{3}\end{array}$ \\
\hline Creatinina al diagnóstico & $1.12 \mathrm{mg} / \mathrm{dl}$ & $0.81 \mathrm{mg} / \mathrm{dl}$ & $1.06 \mathrm{mg} / \mathrm{dl}$ \\
\hline Creatinina de seguimiento & $1.35 \mathrm{mg} / \mathrm{dl}$ & $0.91 \mathrm{mg} / \mathrm{dl}$ & $1.25 \mathrm{mg} / \mathrm{dl}$ \\
\hline $\begin{array}{l}\text { Hallazgos } \\
\text { electrocardiográficos }\end{array}$ & Ritmo sinusal, sin otros hallazgos & $\begin{array}{l}\text { Bloqueo de rama derecha del } \\
\text { haz de His }\end{array}$ & Ritmo de fibrilación auricular \\
\hline $\begin{array}{l}\text { Hallazgos } \\
\text { ecocardiográficos }\end{array}$ & $\begin{array}{l}\text { ETT: VI tamaño normal, FEVI 65\% } \\
\text { con contractilidad normal, patrón } \\
\text { restrictivo mitral } \\
\text { AI muy dilatada, área } 24 \mathrm{~cm}^{2} \text { y } \\
\text { volumen } 52 \mathrm{ml} / \mathrm{m}^{2} \\
\text { Insuficiencia mitral grado II y } \\
\text { aórtica grado I-II } \\
\text { Masa ecogénica (densidad mayor } \\
\text { de tejido blando) en primera } \\
\text { instancia sobre borde endocárdico } \\
\text { segmentos medio y apical en VI, } \\
\text { contráctil, compatible con fibrosis } \\
\text { endomiocárdica } \\
\text { Presión sistólica de la arteria } \\
\text { pulmonar } 83 \text { mmHg } \\
\text { Score de Mucumbi: tres criterios } \\
\text { mayores (8 puntos) y dos } \\
\text { menores (4 puntos); clasificación } \\
\text { de la enfermedad: moderada }\end{array}$ & $\begin{array}{l}\text { ETT: VI tamaño normal, zona de } \\
\text { realce apical sugestiva de fibrosis } \\
\text { endomiocárdica, FEVI } 60 \% \\
\text { Parámetros de función } \\
\text { diastólica anormal con patrón } \\
\text { restrictivo } \\
\text { Al muy dilatada, área } 45 \mathrm{~cm}^{2} \text { y } \\
\text { volumen } 83 \mathrm{ml} / \mathrm{m}^{2} \\
\text { AD dilatada, área } 45 \mathrm{~cm}^{2} \text { y } \\
\text { volumen } 107 \mathrm{ml} / \mathrm{m}^{2} \\
\text { Válvula mitral con insuficiencia } \\
\text { excéntrica moderada } \\
\text { Válvula tricuspídea y pulmonar } \\
\text { con insuficiencia moderada } \\
\text { Derrame pericárdico moderado } \\
\text { Presión sistólica de la arteria } \\
\text { pulmonar } 63 \mathrm{mmHg} \text {, velocidad } \\
\text { regurgitación } 352 \mathrm{~cm} / \mathrm{s} \\
\text { Score de Mucumbi: dos } \\
\text { criterios mayores (6 puntos) y } \\
\text { dos menores (4 puntos); } \\
\text { clasificación de la enfermedad: } \\
\text { moderada }\end{array}$ & $\begin{array}{l}\text { ETT: VI tamaño normal, FEVI } \\
55 \% \\
\text { Al muy dilatada, área } 40 \mathrm{~cm}^{2} \\
\text { y volumen } 99 \mathrm{ml} / \mathrm{m}^{2} \\
\text { AD muy dilatada, área } 40 \mathrm{~cm}^{2} \\
\text { y volumen } 98 \mathrm{ml} / \mathrm{m}^{2} \\
\text { Válvula mitral, aórtica y } \\
\text { tricuspídea con insuficiencia } \\
\text { moderada } \\
\text { Presión sistólica de la arteria } \\
\text { pulmonar } 47 \mathrm{mmHg} \\
\text { Score de Mucumbi: un } \\
\text { criterio mayor (2 puntos) y } \\
\text { dos menores (4 puntos); } \\
\text { clasificación de la } \\
\text { enfermedad: leve }\end{array}$ \\
\hline Comorbilidad & $\begin{array}{l}\text { Hipertensión arterial } \\
\text { Trastorno afectivo bipolar } \\
\text { Insomnio }\end{array}$ & $\begin{array}{l}\text { Masa pulmonar de etiología } \\
\text { benigna } \\
\text { Fibrilación auricular } \\
\text { permanente } \\
\text { Trastorno afectivo bipolar } \\
\text { Hipertensión pulmonar } \\
\text { Hiperuricemia }\end{array}$ & $\begin{array}{l}\text { SAHOS grave } \\
\text { Fibrilación auricular } \\
\text { permanente } \\
\text { Hipotiroidismo } \\
\text { EPOC Gold B }\end{array}$ \\
\hline
\end{tabular}


Tabla 1. Características clínicas, paraclínicas y de seguimiento de los pacientes con fibrosis endomiocárdica (Continuación)

\begin{tabular}{|c|c|c|c|}
\hline Parámetro & Paciente 1 & Paciente 2 & Paciente 3 \\
\hline $\begin{array}{l}\text { Angiografía coronaria y } \\
\text { cateterismo derecho }\end{array}$ & $\begin{array}{l}\text { Sin lesiones significativas, origen } \\
\text { anatómico de la coronaria derecha } \\
\text { cerca del ostium de la coronaria } \\
\text { izquierda } \\
\text { Presión pulmonar: media } 28 \mathrm{mmHg} \text {, } \\
\text { cuña } 20 \mathrm{mmHg} \text {, RVP } 1.8 \mathrm{U} \text { W, GC } 4.34\end{array}$ & $\begin{array}{l}\text { Arterias coronarias epicárdicas } \\
\text { sin lesiones significativas } \\
\text { Presión pulmonar: media } 41 \\
\text { mmHg, cuña } 28 \text { mmHg, RVP } 6 \text { U } \\
\text { W, GC } 4.15\end{array}$ & No se realizó \\
\hline Biopsia endomiocárdica & $\begin{array}{l}\text { Amplias zonas de engrosamiento } \\
\text { endocárdico, con escasas células } \\
\text { inflamatorias mononucleares, } \\
\text { miocardio con arquitectura } \\
\text { conservada, sin fibrosis, sin } \\
\text { infiltrado } \\
\text { Tinción de rojo negativa } \\
\text { Biopsia: fibrosis endomiocárdica }\end{array}$ & $\begin{array}{l}\text { Fragmentos de tejido } \\
\text { miocárdico con núcleos } \\
\text { hipercromáticos, algunos con } \\
\text { aumento del tamaño; } \\
\text { focalmente, en uno de los } \\
\text { fragmentos se aprecia discreto } \\
\text { aumento del tejido fibroso } \\
\text { subendocárdico corroborado en } \\
\text { la coloración especial de } \\
\text { tricrómico de Masson } \\
\text { No se aprecian inflamación, } \\
\text { granulomas ni depósitos }\end{array}$ & No se realizó \\
\hline
\end{tabular}

AD: aurícula derecha; Al: aurícula izquierda; EPOC: enfermedad pulmonar obstructiva crónica; ETT: ecocardiograma transtorácico; FEVI: fracción de eyección del ventrículo izquierdo; GC: Gasto cardiaco; HCM: Hemoglobina corpuscular media; IMC: índice de masa muscular; NYHA: New York Heart Association; RDW: ancho de distribución eritrocitaria; RVP: resistencias vasculares pulmonares; SAHOS: síndrome de apnea/hipopnea obstructiva del sueño; VCM: volumen corpuscular medio; VI: ventrículo izquierdo.

de deterioro de la clase funcional, disnea de esfuerzo y palpitaciones con evidencia de una falla cardiaca con una fracción de eyección del ventrículo izquierdo (FEVI) del 30\% en el contexto de fibrilación auricular, por lo que se consideró un posible origen arrítmico, realizando un manejo dirigido a la modulación de la arritmia supraventricular. Además, en sus antecedentes tenía historia de una masa pulmonar con estudio histológico que descartó un origen neoplásico, hiperuricemia y trastorno del ánimo (Tabla 1). El paciente, pese a que presentó mejoría en la FEVI, continuaba con hospitalizaciones frecuentes por descompensaciones relacionadas con congestión a pesar de un manejo médico optimo dirigido y titulado por guías. A los 7 años del seguimiento en la clínica de insuficiencia cardiaca tuvo una nueva hospitalización por una descompensación Stevenson B. El estudio ecocardiográfico documentó una zona de realce apical en el ventrículo izquierdo, con FEVI del 60\%, disfunción diastólica en patrón restrictivo y dilatación biauricular grave (Fig. 3). En el score de Mucumbi se encontraron dos criterios mayores (6 puntos) y dos criterios menores (4 puntos), con una clasificación de gravedad de la enfermedad de moderada. De manera inicial se planteó llevar a resonancia magnética cardiaca, pero el paciente portaba un dispositivo cardiaco no compatible (cardiorresincronizador cardiaco con desfibrilador, CRT-D), por lo que se consideró la necesidad de realizar biopsia endomiocárdica, descartando en dicho procedimiento enfermedad coronaria (Tabla 1). En el reporte histopatológico se encontró evidencia compatible y que apoyaba la posibilidad de una fibrosis endomiocárdica.

\section{Discusión}

Presentamos los casos que se han documentado en una clínica de insuficiencia cardiaca de un centro de referencia cardiovascular.

Llamativamente, los pacientes comparten algunas características, como hospitalizaciones frecuentes, dilatación auricular grave, presencia de fibrilación auricular y el característico hallazgo de disfunción diastólica en patrón restrictivo, y las imágenes endocárdicas descritas de manera inicial en dos de los pacientes. Todos tuvieron un score ecocardiográfico de Mucumbi diagnóstico de la enfermedad, con clasificaciones de gravedad entre leve y moderada. La biopsia endomiocárdica se utilizó como procedimiento diagnóstico de gran utilidad en nuestros pacientes.

La fibrosis endomiocárdica o enfermedad de Davies es un trastorno olvidado idiopático que predomina en el trópico y el subtrópico en países en vía de desarroIlo, afectando a poblaciones pobres, que característicamente produce un engrosamiento fibrótico del endocardio y el miocardio en uno o ambos ventrículos, generando un llenado ventricular restrictivo'. Pese a que esta enfermedad fue descrita de manera inicial por Loffler en $1936^{2}$ en un paciente con eosinofilia 
asociada y en 1946 por Bedford y Konstam ${ }^{3}$ en África, sus características clínico-patológicas fueron detalladas por Davies ${ }^{4}$ en Uganda en 1948.

Esta enfermedad es una importante causa de falla cardiaca en África, llegando en algunos lugares (zona rural de Mozambique) a explicar el $20 \%$ de los casos $^{5}$. Aunque su fisiopatología y su etiología no están del todo claras, su patología se asemeja a la de condiciones como la cardiomiopatía eosinofílica y el síndrome hipereosinofílico. Es por ello que esta condición se considera como parte de un proceso patológico único que también incluye la endocarditis de Loffler ${ }^{6}$.

El factor etiológico especifico es desconocido. Hay varias teorías que intentan explicar la geografía y la patología inusual de la enfermedad. La concentración en el trópico ha dirigido las investigaciones a causas infecciosas o nutricionales. La similitud con las lesiones vistas en la endocarditis de Loffler y el síndrome carcinoide cardiaco sugiere un efecto tóxico de la serotonina o eosinofilia; sin embargo, las inconsistencias entre la geografía de estas condiciones es un argumento en contra ${ }^{7}$. Los factores que de manera más frecuente se han implicado en la etiopatogenia de la enfermedad son la etnia, la pobreza, el consumo de mandioca, la serotonina, la malnutrición, la deficiencia de magnesio, cerio y vitamina D, las infecciones (Plasmodium, Schistosoma, microfilaria, helmintos, Coxsackie B, arbovirus, Toxoplasma gondii), la autoinmunidad, la eosinofilia, algunos agentes tóxicos y factores genéticos. La combinación de anormalidades del magnesio y del cerio produce lesiones similares a las de la enfermedad en animales de laboratorio ${ }^{8}$. No se conoce cuál de estos agentes tiene una relación causa-efecto o si funcionan como un disparador de un proceso latente. Un hecho interesante que apoya las anormalidades nutricionales es el dato presentado por Gupta, et al. ${ }^{9}$, demostrando que una dieta enriquecida para prevenir anormalidades del magnesio y del cerio reduce la ocurrencia de la enfermedad. El marcador patológico de la enfermedad es el engrosamiento endocárdico difuso o focal, involucrando ambos ventrículos el $50 \%$ de los casos, y en el $40 \%$ solo se afecta el ventrículo izquierdo. El engrosamiento focal y la fibrosis producen la fisiología restrictiva. La fibrosis se genera por proliferación fibroblástica y depósito de colágeno ${ }^{10}$, que cambia sus componentes e incrementa de manera anormal el tipo I, lo que genera la rigidez miocárdica ${ }^{11}$. En el ventrículo izquierdo, la fibrosis se extiende desde el ápex hasta la valva posterior de la válvula mitral, respetando la valva anterior y el tracto de salida, y en algunas ocasiones comprometiendo el músculo papilar y las cuerdas tendinosas. Hay depósito de tejido de fibrosis denso en el subendocardio, con trombosis y calcificación en estadios avanzados ${ }^{10}$.

Desde el punto de vista epidemiológico, la enfermedad es mas prevalente en África, Asia y Suramérica. En la región subsahariana predomina en Uganda, el cinturón costero de Mozambique y algunas partes del Oeste. En la India se ha documentado un brote en el estado de Kerala, y en China hay una alta prevalencia en la provincia de Guangxi. En Suramérica, los casos han sido reportados en Brasil y Colombia ${ }^{12}$. El único estudio realizado en Mozambique encontró que una de cada cinco personas tenía fibrosis endomiocárdi$\mathrm{ca}^{13}$. Los mayores determinantes son la edad, el sexo, la etnia, la deprivación social y la eosinofilia. Mas de la mitad de los casos se diagnostican en la primera década de la vida, con un segundo pico de incidencia en mujeres en edad fértili,12. La eosinofilia asociada es un factor primario para la enfermedad; un hallazgo no explicado por infecciones parasitarias ${ }^{14}$. En los últimos años se ha notado una disminución de la incidencia de la enfermedad, posiblemente por una mejoría en los estándares de vida con decline de la malnutrición, las infecciones, la infestación parasitaria y la eosinofilia ${ }^{15}$.

La evolución natural no se entiende por completo debido a la presentación tardía de los pacientes en búsqueda de atención médica. Típicamente hay un inicio insidioso, con síntomas de presentación relacionados con la cámara o la válvula que más se compromete, la duración de la enfermedad y los signos de actividad. La fase inicial es la fase de carditis, en la que destaca la enfermedad febril y en casos graves puede ocurrir choque cardiogénico. Los pacientes que sobreviven progresan a la fase subaguda o crónica. Los síntomas más frecuentes se relacionan con hipertensión venosa sistémica crónica, como ingurgitación yugular, hepatomegalia y esplenomegalia. En el compromiso bilateral hay una combinación de signos clásicos de falla cardiaca ${ }^{5}$.

Las complicaciones como fibrilación auricular, eventos tromboembólicos e insuficiencia auriculoventricular progresiva son las que se reportan ${ }^{5}$. Pese al diagnóstico tardío en nuestros pacientes, estos presentaban ya fibrilación auricular, insuficiencia valvular y eventos tromboembólicos. Si bien menos del $50 \%$ de los pacientes presentan ascitis, esta generalmente refleja un mayor compromiso del ventrículo derecho, más duración de la enfermedad y peor pronóstico ${ }^{16}$. Otros factores relacionados con mayor mortalidad en este grupo de pacientes son el compromiso biventricular, la 


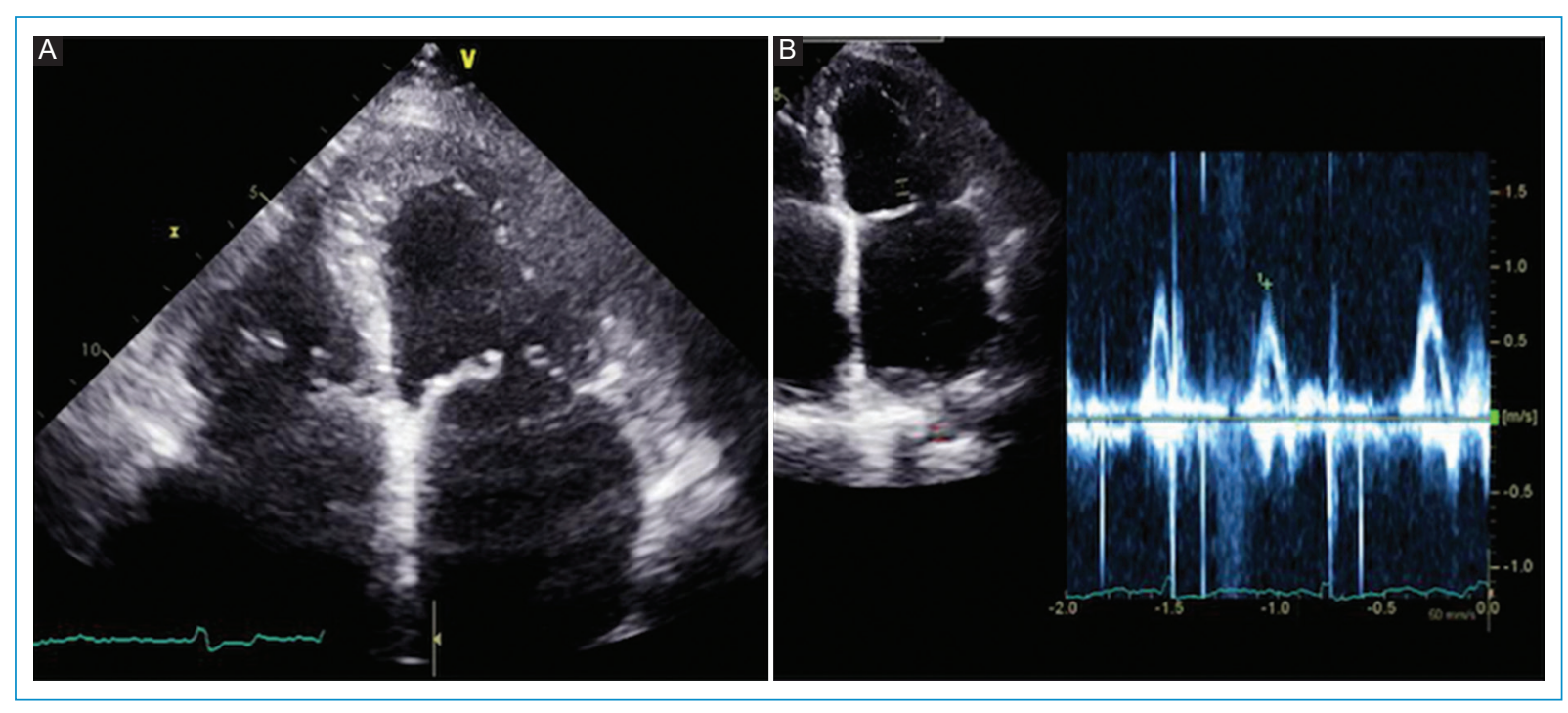

Figura 3. Ecocardiograma del caso 3. A: vista apical de cuatro cámaras donde se aprecia una importante dilatación biauricular con un ventrículo de tamaño normal sin anormalidad endocárdica, pero con un aumento leve del realce a nivel del segmento basal de la pared inferoseptal. B: influjo mitral que muestra una sola onda por ritmo de fibrilación auricular durante el estudio.

fibrosis del ventrículo derecho y la insuficiencia mitral y tricuspídea asociada ${ }^{5,17}$.

A partir del estudio de Mucumbi se realizó una escala útil para establecer el diagnóstico y también predecir el pronóstico que se debe considerar en estos pacientes ${ }^{13}$. Los hallazgos electrocardiográficos a menudo sugieren anormalidades de la aurícula derecha, con alteraciones de la onda $\mathrm{P}$ en la derivada DIl y un patrón de QR con $\mathrm{R}$ diminuta en $\mathrm{V} 1$ cuando predomina el compromiso del ventrículo derecho ${ }^{18}$. La fibrilación auricular es frecuente, en especial cuando hay compromiso del ventrículo derecho, y se asocia a mayor riesgo de falla cardiaca y peor pronóstico ${ }^{17}$. Otros hallazgos son bloqueos cardiacos o ritmos de la unión, desviación del eje a la derecha y anormalidades de la conducción intraventricular ${ }^{19}$. El ecocardiograma es fundamental en el proceso diagnóstico, con el hallazgo de obliteración del ápex con dilatación auricular siendo altamente sugestivo. El diagnóstico es definido por la identificación de fibrosis en las cámaras ventriculares, signos de restricción de llenado ventricular y signos de compromiso de las válvulas auriculoventriculare ${ }^{20}$; estos hallazgos estaban presentes en todos los pacientes con un score de Mucumbi diagnóstico. La resonancia desempeña un papel en el diagnóstico y el pronóstico, pues proporciona una evaluación morfológica precisa, con características restrictivas típicas de ventrículos pequeños o no dilatados, con elementos de hipertrofia apical. También hay un aumento típico de la aurícula por la grave disfunción diastólica, con patrón restrictivo y realce subendocárdico que se extiende desde la región subvalvular a los ápices de los dos ventrículos; los trombos se describen con frecuen$\mathrm{cia}^{21}$. Salemi, et al. ${ }^{22}$ publicaron un interesante estudio de resonancia con confirmación histológica de fibrosis endomiocárdica. Ellos documentaron que la distribución del realce tardío con gadolinio se correspondió con tejido fibrótico comúnmente extendiéndose desde la región subvalvular al ápex de los ventrículos, donde era más prominente. Una apariencia de tres capas fue típica en esta enfermedad: una capa interna sin perfusión por la presencia de trombos, una capa media que mostró realce tardío con gadolinio por la fibrosis y una capa externa de miocardio normal; no se presentan los datos de resonancia por las imposibilidades mencionadas para realizarla en este grupo de pacientes ${ }^{22}$. El papel de la biopsia endomiocárdica es controversial en este tipo de pacientes $^{23}$. Aunque este procedimiento puede ser redundante en estadios avanzados, su aplicación en pacientes asintomáticos o con síntomas leves y hallazgos ambiguos en los demás estudios de imágenes puede ayudar a obtener un correcto diagnóstico, a la vez que se excluyen otras causas de cardiomiopatía restrictiva. Los hallazgos histológicos son un engrosamiento fibroso endocárdico extenso compuesto de fibras de colágeno con cambios estructurales con predominancia de las de tipo I sobre las de tipo III, y proliferación de vasos pequeños, situación importante para el mantenimiento de la fibrosis ${ }^{24}$; el infiltrado inflamatorio en general es de 


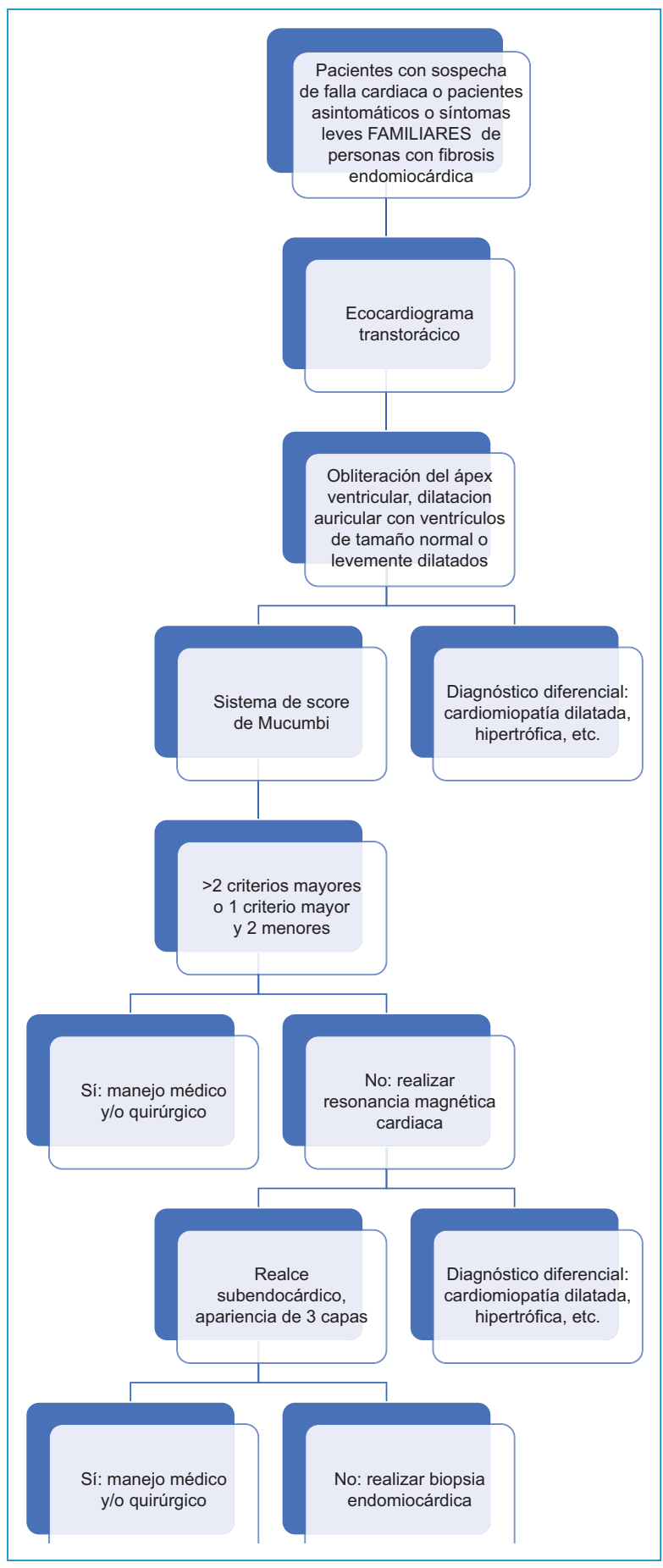

Figura 4. Algoritmo diagnóstico propuesto para pacientes con sospecha de fibrosis endomiocárdica.

leve a moderado, con predominio de macrófagos y linfocitos $\mathrm{T}^{10}$. En nuestra consideración, y según la disponibilidad de recursos en nuestro medio, los pacientes deberían abordarse con el algoritmo de trabajo que se muestra en la figura 4.
Infortunadamente, no hay tratamiento efectivo o específico que mejore la supervivencia de estos pacientes. El manejo sintomático con diuréticos y vasodilatadores es importante para mejorar la calidad de vida. Los pacientes con síntomas graves deben ser considerados para terapia quirúrgica, pues solo el manejo médico tiene poca efectividad. En general, se requieren operadores experimentados y un equipo de manejo posquirúrgico familiarizado con los cuidados de pacientes complejos. La cirugía se recomienda para todos los pacientes con clase funcional III o IV de la New York Heart Association (NYHA) ${ }^{25,26}$, y debe realizarse antes que haya un daño hepático o cardiaco irreversible. Los pacientes con ascitis de larga evolución, caquexia extrema, tromboembolia pulmonar crónico, fibrosis o calcificación endocárdica extensa, función ventricular alterada o acortamiento extremo de las valvas, representan pacientes quirúrgicos de alto riesgo y tienen una contraindicación quirúrgica relativa $^{27}$. La endocardectomía y el reparo o reemplazo es la estrategia quirúrgica mas común. En muchos pacientes, un plano relativamente bien definido de corte permite la remoción del endocardio fibrótico y la preservación del miocardio sano. El reparo valvular se favorece sobre el reemplazo mecánico debido a los retos del manejo de anticoagulación ${ }^{12}$. La reducción atrial se realiza de manera concurrente para disminuir el riesgo de arritmias o trombos atriales ${ }^{28}$. La anastomosis cavopulmonar y el reparto en «ventrículo 1.5» pueden ayudar en el manejo paliativo de pacientes con una fibrosis endomiocárdica de compromiso predominante derecho grave, como también se ha utilizado para estabilizar a los pacientes antes y después de la endocardectomía cuando la cavidad ventricular derecha permanece de un tamaño inadecuado ${ }^{29,30}$. La mortalidad posquirúrgica es del $18-29 \%$ y típicamente se relaciona con complicaciones como síndrome de bajo gasto cardiaco, taponamiento y derrame pericárdico, como bloqueo auriculoventricular completo $25,31,32$. En una serie de 83 pacientes en Brasil que sufrieron manejo quirúrgico, la supervivencia a los 17 años fue del $55 \% 25$

\section{Conclusiones}

La fibrosis endomiocárdica es una enfermedad olvidada de las regiones tropicales relacionada con factores como la pobreza y los bajos recursos, y con diagnóstico tardío, lo que impacta en el pronóstico de estos pacientes. Debe considerarse en pacientes con 
falla cardiaca de inicio temprano con un patrón restrictivo asociado, en el contexto de las complicaciones típicas (fibrilación auricular, insuficiencia de válvulas auriculoventriculares) y con poca respuesta al manejo farmacológico convencional.

\section{Financiamiento}

Está investigación no recibió financiamiento por parte de entidades públicas o privadas para su realización.

\section{Conflicto de intereses}

Los autores manifiestan no tener conflictos de intereses para el presente estudio.

\section{Responsabilidades éticas}

Protección de personas y animales. Los autores declaran que para esta investigación no se han realizado experimentos en seres humanos ni en animales.

Confidencialidad de los datos. Los autores declaran que han seguido los protocolos de su centro de trabajo sobre la publicación de datos de pacientes.

Derecho a la privacidad y consentimiento informado. Los autores declaran que en este artículo no aparecen datos de pacientes.

\section{Bibliografía}

1. Mocumbi AO, Yacoub S, Yacoub MH. Neglected tropical cardiomyopathies: II. Endomyocardial fibrosis: myocardial disease. Heart Br Card Soc. 2008;94:384-90.

2. Knorr D, Scheppe KJ. [Endocarditis parietalis fibroplastica with eosinophilia (Loeffler's) in a 7-year-old child; first description in a child]. Z Kinderheilkd. 1958;81:102-12.

3. Bedford DE, Konstam GLS. Heart failure of unknown aetiology in Africans. Br Heart J. 1946;8:236.

4. Davies JNP. Endomyocardial fibrosis in Uganda. East Afr Med J. 1948;25:10.

5. Duraes AR, de Souza Lima Bitar Y, Roever L, Neto MG. Endomyocardial fibrosis: past, present, and future. Heart Fail Rev. 2020;25:725-30.

6. Kumar P, Chandrashekhara SH, Kumar S, Malhi AS. Loeffler's endocarditis with isolated left ventricular involvement on cardiac MRI. BMJ Case Rep. 2019;12:e227642.

7. Bukhman G, Ziegler J, Parry E. Endomyocardial fibrosis: still a mystery after 60 years. PLoS Negl Trop Dis. 2008;2:e97.

8. Mocumbi AO. Endomyocardial fibrosis: a form of endemic restrictive cardiomyopathy. Glob Cardiol Sci Pract. 2012;2012:11.
9. Gupta PN, Kunju SM, Rajan B, Koshy AG, Vishwanathan S, George PS, et al. Geographical variation in the clinical presentation of endomyocardial fibrosis in India? Indian Heart J. 2018;70:56-65.

10. Iglezias SD, Benvenuti LA, Calabrese F, Salemi VMC, Silva AMG, Carturan $E$, et al. Endomyocardial fibrosis: pathological and molecular findings of surgically resected ventricular endomyocardium. Virchows Arch Int J Pathol. 2008;453:233-41.

11. Radhakumary C, Kumari TV, Kartha CC. Endomyocardial fibrosis is associated with selective deposition of type I collagen. Indian Heart $\mathrm{J}$. 2001;53:486-9.

12. Grimaldi A, Mocumbi AO, Freers J, Lachaud M, Mirabel M, Ferreira B, et al. Tropical endomyocardial fibrosis: natural history, challenges, and perspectives. Circulation. 2016;133:2503-15.

13. Mocumbi AO, Ferreira MB, Sidi D, Yacoub MH. A population study of endomyocardial fibrosis in a rural area of Mozambique. $\mathrm{N}$ Engl J Med. 2008;359:43-9.

14. Rutakingirwa M, Ziegler JL, Newton R, Freers J. Poverty and eosinophilia are risk factors for endomyocardial fibrosis (EMF) in Uganda. Trop Med Int Health TM IH. 1999;4:229-35.

15. Vijayaraghavan G, Sivasankaran S. Tropical endomyocardial fibrosis in India: a vanishing disease! Indian J Med Res. 2012;136:729-38.

16. Barretto ACP, Mady C, Oliveira SA, Arteaga E, Dal Bo C, Ramires JAF. Clinical meaning of ascites in patients with endomyocardial fibrosis. Arq Bras Cardiol. 2002;78:196-9.

17. Barretto AC, Mady C, Nussbacher A, Ianni BM, Oliveira SA, Jatene A, et al. Atrial fibrillation in endomyocardial fibrosis is a marker of worse prognosis. Int J Cardiol. 1998;67:19-25.

18. Tharakan JA. Electrocardiogram in endomyocardial fibrosis. Indian Pacing Electrophysiol J. 2011;11:129-33.

19. Basile U, Carlisle R, Falase AO. E.C.G. in endomyocardial fibrosis. East Afr Med J. 1974;51:444-8.

20. Tello R, Cuan V, Abundes A, Navarro J, García Lara J, Astudillo R, et al. [Doppler echocardiography in endomyocardial fibrosis]. Arch Inst Cardiol Mex. 1994;64:251-5.

21. Kharabish A, Haroun D. Cardiac MRI findings of endomyocardial fibrosis (Loeffler's endocarditis) in a patient with rheumatoid arthritis. J Saudi Heart Assoc. 2015;27:127-31.

22. Salemi VMC, Rochitte CE, Shiozaki AA, Andrade JM, Parga JR, de Ávila LF, et al. Late gadolinium enhancement magnetic resonance imaging in the diagnosis and prognosis of endomyocardial fibrosis patients. Circ Cardiovasc Imaging. 2011;4:304-11.

23. Barretto AC, Bellotti G, Higuchi M de L, Stolf NA, Dauar D, Mady C, et al. [Endomyocardial biopsy of the right ventricle in patients with endomyocardial fibrosis]. Arq Bras Cardiol. 1986;46:19-21.

24. Davies JN, Ball JD. The pathology of endomyocardial fibrosis in Uganda. Br Heart J. 1955;17:337-59.

25. Moraes F, Lapa C, Hazin S, Tenorio E, Gomes C, Moraes CR. Surgery for endomyocardial fibrosis revisited. Eur J Cardiothorac Surg. 1999:15:309-12; discussion 312-3.

26. Mocumbi AO, Carrilho C, Burke MM, Wright G, Yacoub MH. Emergency surgical treatment of advanced endomyocardial fibrosis in Mozambique. Nat Clin Pract Cardiovasc Med. 2009;6:210-4.

27. Bertrand E, Chauvet J, Assamoi MO, Charles D, Ekra E, Dienot BB, et al. Results, indications and contra-indications of surgery in restrictive endomyocardial fibrosis: comparative study on 31 operated and 30 non-operated patients. East Afr Med J. 1985;62:151-60.

28. Mocumbi AO, Sidi D, Vouhe P, Yacoub M. An innovative technique for the relief of right ventricular trabecular cavity obliteration in endomyocardial fibrosis. J Thorac Cardiovasc Surg. 2007;134:1070-2.

29. Yie K, Sung S, Kim D, Woo J. Bidirectional cavopulmonary shunt as a rescue procedure for right ventricular endomyocardial fibrosis. Interact Cardiovasc Thorac Surg. 2004;3:86-8.

30. Mishra A, Krishna Manohar SR, Sankar Kumar R, Valiathan MS. Bidirectional Glenn shunt for right ventricular endomyocardial fibrosis. Asian Cardiovasc Thorac Ann. 2002;10:351-3.

31. da Costa FD, Moraes CR, Rodriques JV, de Mendonça JT, de Andrade JC, Buffolo $E$, et al. Early surgical results in the treatment of endomyocardial fibrosis. A Brazilian cooperative study. Eur J Cardiothorac Surg. 1989;3:408-13.

32. Valiathan MS, Balakrishnan KG, Sankarkumar R, Kartha CC. Surgical treatment of endomyocardial fibrosis. Ann Thorac Surg. 1987;43:68-73. 